\title{
Human Osteoblast Growth and Maturation in Response to Metformin and the Thienopyridone, A769662
}

\author{
Blackburn $\mathrm{J}^{1}$, Burston $\mathrm{BJ}^{1}$, Shepherd $\mathrm{S}^{2}$, Bone $\mathrm{H}^{2}$, Blom AW ${ }^{1}$ and Mansell $\mathrm{JP}^{2 *}$ \\ ${ }^{1}$ Musculoskeletal Research Unit, University of Bristol, Southmead Hospital, Westbury on Trym, Bristol, UK \\ ${ }^{2}$ CATIM laboratory, University of the West of England, Coldharbour Lane, Bristol, UK
}

*Corresponding author: Mansell JP, CATIM laboratory, University of the West of England, Coldharbour Lane, Bristol, BS16 1QY, UK, Tel: +44 117328 3573, Fax: +44 117328 2132, E-mail: jason.mansell@uwe.ac.uk

Citation: Blackburn J, Burston BJ, Shepherd S, Bone H, Blom AW, et al. (2014) Human Osteoblast Growth and Maturation in Response to Metformin and the Thienopyridone, A769662. SAJ Pharm Pharmacol 1: 102. doi: $10.18875 / 2375-2262.1 .102$

Article history: Received: 29 May 2014, Accepted: 16 June 2014, Published: 14 August 2014

\begin{abstract}
Metformin (Met) is a biguanide drug widely used in the treatment and management of non insulin-dependent diabetes mellitus. In recent years it has emerged that Met, by stimulating adenosine monophosphate-activated protein kinase (AMPK), can promote the maturation of osteoblasts, albeit cells sourced from rodent and murine calvaria. Finding novel uses for existing drugs is especially appealing, primarily from the fiscal and time constraints posed in developing new products. Identifying agents capable of supporting human osteoblast growth and differentiation are attractive in a bone regenerative context. Since studies using Met are invariably restricted to rodent and murine osteoblasts we sought to investigate whether this biguanide might have a positive influence upon human osteoblast growth and maturation. To this end we examined the effect of Met on two osteoblast-like cell lines, MG63 and Saos-2, and compared the responses to primary human osteoblasts and their bone marrow-derived stem cell progeny. Furthermore we examined the effect of a cell permeable Met surrogate, A769662, which is a potent and far more selective activator of AMPK. Herein we report that Met is without influence on cell growth. Furthermore the application of Met, albeit in the millimolar range, actually inhibited osteoblast maturation. Conversely A769662 was toxic to the osteosarcoma-derived cell lines, MG63 and Saos-2, but without effect on the growth of primary cells or their stem cell progenitors. Since the cell lines are known to be p53 deficient we propose that activation of AMPK by A769662 could form part of the arsenal in the fight against osteosarcoma.
\end{abstract}

Keywords: Metformin; A769662; Osteoblast; Osteosarcoma

\section{Introduction}

Small molecules with the potential to promote human osteoblast maturation are desirable in a bone regenerative context, particularly if they have proven efficacy in disease management or treatment; finding novel uses for existing drugs saves time and costs for drug development and is a proven short cut between laboratory and clinic. Indeed James Black (pharmacologist and Nobel laureate) reminds us that "the most fruitful basis for the discovery of a new drug is to start with an old drug" [1,2]. A potentially interesting candidate that might fulfil this role is the biguanide metformin (Met). Originally sold as Glucophage, Met is a first-line drug of choice for the treatment of type 2 diabetes [3] but there is emerging evidence that Met may have beneficial effects on bone by supporting osteoblast maturation. Thus far all the studies exploring the action of Met on osteoblast activity have looked at murine or rodent cells sourced from ectodermally derived calvarial tissue, Met has been found to promote collagen and alkaline phosphatase (ALP) expression in rat and mouse osteoblasts [4] and to enhance murine osteoblast differentiation, as supported by raised matrix calcification in extended cell cultures [5-7]. In addition, Met has been reported to attenuate osteopaenia in ovariectomised rats [8] and to support an osteogenic response in rodent bone marrow derived stromal cells [9]. The largely held view from these collected works is that Met influences osteoblast activity via its ability to stimulate adenosine monophosphate-activated protein kinase (AMPK), a heterotrimeric protein central to maintaining cellular energy homeostasis $[10,11]$. Suffice it to say conditions leading to energy depletion, such as exercise, activate AMPK [12] resulting in the suppression of energy consumption, e.g., gluconeogenesis, but activation of energy generation, e.g., glycolysis. 
Importantly the skeleton is now believed to directly participate in energy metabolism by modulating glucose homeostasis [13], developments which initially arose through an osteocalcin (OC) knockout murine model [14]. OC is an abundant non-collagenous component of bone tissue and was believed to be implicated in bone matrix calcification. However OC deficient mice have no skeletal phenotype at birth but do show signs of bone accrual with maturity coupled with increased visceral fat, insulin resistance and glucose intolerance. In more recent times it has been suggested that OC might be a link between obesity and insulin resistance [15], a postulation founded on the finding that OC regulates insulin secretion [16]. These recent revelations, which point to the possibility of a bone-pancreas endocrine loop in whole body energy homeostasis, have forged the basis of topical perspectives by leaders in the field $[17,18]$.

In light of the evidence presented it would appear that Met has potential for orthopaedic application but studies using human osteoblasts and their bone marrow progenitors have not been forthcoming. Since our ongoing programme of research is targeted towards enhancing the experience of arthroplasty this necessitates an examination of human osteoblasts which have their origins with the mesoderm. To this end we have focussed on the MG63 osteoblast-like cell line, primary human osteoblasts (hOBs) and their marrow derived mesenchymal stem cell progenitors (hBMSCs).

\section{Materials \& Methods}

\section{Cell culture materials}

Unless stated otherwise all reagents were from Sigma (Poole, UK). Tissue culture medium ( $\alpha$-MEM \& DMEM:Hams F12), fetal calf serum (FCS) and Trypsin-EDTA were obtained from Invitrogen (Paisley, Scotland) and stored at $4{ }^{\circ} \mathrm{C}$. Both of these media were supplemented with L-glutamine (final concentration of $4 \mathrm{mM}$ ), sodium pyruvate (final concentration of $1 \mathrm{mM}$ ), penicillin $(0.1$ units $/ \mathrm{mL}$ ), streptomycin (100ng/mL), a 100-fold dilution of a non-essential amino acid stock (Sigma cat\# M7145) and FCS (10\% $\mathrm{v} / \mathrm{v})$. Metformin $(20 \mathrm{mM})$ and phenformin $(10 \mathrm{mM})$ were prepared within minutes of use in serum-free tissue culture media and $0.2 \mathrm{uM}$ filter sterilised prior to treating cultured cells. The lysophosphatidic acid (LPA) receptor agonist, (3S) 1-fluoro-3-hydroxy4-butyl-1-phosphonate (FHBP, tebu-bio, Peterborough, UK) was prepared as a 1mM stock in 1:1 ethanol:tissue culture grade water and stocks of $1 a, 25$-dihydroxyvitamin D3 (D3, 100 $\mu \mathrm{M})$ prepared in ethanol and stored at $-20{ }^{\circ} \mathrm{C}$. 5-aminoimidazole-4-carboxamide-1-b-D-ribofuranoside (AiCAR) was prepared fresh in serum-free culture medium (SFCM) up to 2.5mM. The AMPK activator, A769662 (Tocris, Bristol, UK) was prepared as a 100mM stock in DMSO and stored at - $20^{\circ} \mathrm{C}$. The AMPK inhibitor, (6-[4-(2-Piperidin-1-yl-ethoxy)-phenyl)]-3-pyridin-4-yl-pyyrazolo[1,5-a] pyrimidine (Compound C) and a cell permeable broad spectrum caspase inhibitor, N-(2-Quinolyl)valyl-aspartyl-(2,6-difluorophenoxy)methyl Ketone (Q-VD, Merck Chemicals Ltd. Nottingham, UK) were reconstituted to $10 \mathrm{mM}$ in DMSO and kept at $-20{ }^{\circ} \mathrm{C}$.

\section{Osteoblast cultures and stimulations}

The human osteoblast cell lines, MG63 and Saos-2 and commercial primary human osteoblasts (hOBs, Promocell, Heidelberg, Germany) were used. Each cell type was grown to confluency under conventional culturing conditions in supplemented DMEM:Hams F12. Having reached confluency the cells were harvested using trypsin-EDTA, resuspended in fresh medium and dispensed into 24-well culture plates (Greiner Bio One) at a density of $20 \mathrm{~K}$ cells $/ \mathrm{ml} /$ well. In the first instance we examined the potential of Met to influence MG63 maturation alongside our established model of differentiation [19] which involves costimulating MG63s with D3 (100nM) and an LPA receptor agonist, in this case FHBP (500nM). Briefly, after three days of culture the medium was removed and the cells treated with SFCM for 24 hours prior to SFCM alone (control) or SFCM supplemented with D3 (100nM), FHBP (500nM), their combination, either alone or in the presence of Met (1.25-10mM). Subsequent studies were performed using AiCAR (2.5mM), Phen (0.5-5mM) or A769662 (25-100 $\mathrm{MM})$. An assessment of osteoblast growth and maturation were determined after 2-3 days. In some instances the cells were exposed to inhibitors in an attempt to identify mechanisms of compound action.

\section{Human bone marrow derived stem cell (hBMSC) treatments}

This study utilised hBMSCs sourced commercially (Promocell, Heidelberg, Germany, lot no: 0051105.11) and characterised as being $>95 \%$ positive for CD44 and CD105 and >95\% negative for CD31 and CD45. In addition these same cells were screened for their ability to form the osteoblast phenotype in response to standard osteogenic stimuli, i.e., ascorbate, BGP and DEX with a differentiating capacity of more than 10 population doublings. Cells were directly placed into $75 \mathrm{~cm}^{2}$ flasks containing $\alpha-\mathrm{MEM}$ supplemented with $10 \%(\mathrm{v} / \mathrm{v})$ FCS and allowed to reach confluency ( 1 week) prior to any experimentation or storage under liquid nitrogen. For experiments hBMSCs were seeded at a density of $20 \mathrm{~K} / \mathrm{ml} /$ well into 24 -well tissue culture plates and treated exactly as described for the osteoblasts in the previous section.

\section{Cell proliferation assay and determination of cell number}

An assessment of cell number was performed using a combination of the tetrazolium compound 3-(4,5-dimethylthiazol-2-yl)-5(3-carboxymethoxy-phenyl)-2-(4-sulfophenyl)-2H-tetrazolium, innersalt (MTS, Promega, UK) and the electron coupling reagent phenazine methosulphate (PMS). Each compound was prepared separately in pre-warmed $\left(37^{\circ} \mathrm{C}\right)$ phenol red free DMEM/F12, allowed to dissolve and then combined so that $1 \mathrm{~mL}$ of a $1 \mathrm{mg} / \mathrm{mL}$ solution of PMS was combined to $19 \mathrm{~mL}$ of a $2 \mathrm{mg} / \mathrm{mL}$ solution 
of MTS. A stock suspension of MG63s $\left(1 \times 10^{6}\right.$ cells $\left./ \mathrm{mL}\right)$ was serially diluted in the same growth medium to give a series of known cell concentrations down to $25 \times 10^{3}$ cells $/ \mathrm{mL}$. Each sample $(0.5 \mathrm{~mL}$ in a microcentrifuge tube) was spiked with $0.1 \mathrm{~mL}$ of the MTS/ PMS reagent mixture and left for 45 min within a tissue culture incubator. A blank consisted of media alone $(0.5 \mathrm{~mL})$ plus $0.1 \mathrm{~mL}$ of the MTS/PMS reagent mixture. Once incubated, the samples were centrifuged at $900 \mathrm{rpm}$ to pellet the cells and $0.1 \mathrm{~mL}$ of the supernatants dispensed onto a 96 -well microtitre plate and the absorbances read at $492 \mathrm{~nm}$ using a multiplate reader. Plotting the absorbances against known cell number, as assessed initially using haemocytometry, enabled extrapolation of cell numbers for the experiments described herein.

In evaluating the extent of cell growth for the multi-well plates the test media was removed and replaced with $0.5 \mathrm{~mL}$ of phenol red free DMEM/F12 and each well subsequently spiked with $0.1 \mathrm{~mL}$ of the MTS/PMS reagent. Plates were returned to the incubator and left for $45 \mathrm{~min}$ under conventional culturing conditions. Once incubated, samples $(0.1 \mathrm{~mL})$ from each well were dispensed onto a 96-well microtitre plate and the absorbances at $492 \mathrm{~nm}$ read using a multiplate reader. Plates were staggered to ensure that all samples were recovered for 96-well plating within 5 min to minimise any error introduced during the formation of further formazan product by the cell monolayer.

\section{Total alkaline phosphatase (ALP) activity}

An assessment of ALP activity is reliably measured by the generation of p-nitrophenol (p-NP) from p-nitrophenylphosphate (p-NPP) under alkaline conditions. The treatment of cells to quantify ALP activity was similar to that described by us recently $[20,21]$. Briefly the remaining MTS/PMS reagent was removed and the monolayers incubated for a further 5 min in fresh phenol red-free DMEM/F12 to flush out the residual formazan. Following this incubation period the medium was removed and the monolayers lysed with $0.1 \mathrm{~mL}$ of $25 \mathrm{mM}$ sodium carbonate ( $\mathrm{pH} 10.3), 0.1 \%(\mathrm{v} / \mathrm{v})$ Triton X-100. After 2 min each well was treated with $0.2 \mathrm{~mL}$ of $15 \mathrm{mM} \mathrm{p}$-NPP (di-tris salt, Sigma, UK) in $250 \mathrm{mM}$ sodium carbonate (pH 10.3), $1.5 \mathrm{mM} \mathrm{MgCl}_{2}$. Lysates were then left under conventional cell culturing conditions for $1 \mathrm{~h}$. After the incubation period, $0.1 \mathrm{~mL}$ aliquots were transferred to a 96 -well microtitre plate and the absorbance read at $405 \mathrm{~nm}$. An ascending series of p-NP $(25-400 \mu \mathrm{M})$ prepared in the incubation buffer enabled quantification of product formation.

\section{Assessment of cell morphology}

Chamber slides, four-well (Lab-Tek Chamber Slide systems, Nalge Nunc International, Roskilde, Denmark), were seeded with 1 $\mathrm{ml} /$ well of a $20 \mathrm{~K}$ cells $/ \mathrm{ml}$ suspension and the cells left, as described above, for 24 hours. After this initial culture period the medium was removed and the cells treated with either SFCM, or SFCM supplemented with 50 or $100 \mu \mathrm{M}$ A769662 for a further 24 and 48 hours. Following the incubation period cells were processed for F-actin detection using the fungal toxin phalloidin conjugate, Alexa Fluor $594^{\mathrm{R}}$ (Invitrogen, reconstituted to 200 units $/ \mathrm{ml}$ in methanol, stored at $-20^{\circ} \mathrm{C}$ ). Briefly, the culture media was removed and the cells rinsed once with PBS and then fixed with 2.5\% glutaraldehyde in PBS for $5 \mathrm{~min}$ at room temperature. The fixative was then aspirated and the cells rinsed twice with PBS before treatment with $0.25 \mathrm{ml} /$ well Alexa Fluor $594^{\mathrm{R}}$ prepared as a 40 fold dilution in PBS and left for $5 \mathrm{~min}$, in the dark, at room temperature. After the incubation period, wells were aspirated and rinsed twice with PBS prior to treatment with DAPI slide mountant (Vectashield, Vector Laboratories, Peterborough, UK). Cells were viewed using an Olympus BX14 microscope equipped with single band pass filters for the visualisation of nuclei (excitation $355 \mathrm{~nm} /$ emission $465 \mathrm{~nm}$ ) and F-actin (excitation 550nm/emission 570nm). Images were captured using Metasystems ISIS software. In other instances images of cells grown within 24-well plates were taken as viewed with a standard inverted light microscope fitted with a digital camera (Zeiss) and the images processed within Paintshop Pro operated from a PC.

\section{Statistical analysis}

Unless stated otherwise, all experiments described were performed three times and all data were subject to a one-way analysis of variance (ANOVA) to test for statistical significant. When a $p$ value of $<0.05$ was found, a Tukey multiple comparisons post-test was performed between all groups.

\section{Results}

\section{Metformin and AiCAR inhibit the maturation response of MG63 osteoblasts}

In an initial series of experiments we exposed MG63 cells to a wide range of Met concentrations (10 $\mu \mathrm{M}-10 \mathrm{mM})$ to ascertain if this biguanide might influence cell growth and/or maturation. In isolation Met had no influence on either parameter (data not shown). Next we considered whether Met might influence the differentiation response to cells treated with a proven ligand combination known to precipitate a demonstrable maturation event. To this end MG63s were co-stimulated with D3 (100nM) and an LPA1 receptor agonist, FHBP $(500 \mathrm{nM})$, in the presence and absence of Met (1.25-10mM). As expected the co-treatment of MG63 cells with D3 and FHBP led to a stark increase in $\mathrm{p}$-NP from $\mathrm{p}$-NPP (Figure 1A) reflecting greater total ALP activity $\left({ }^{*} \mathrm{p}<0.0001\right.$ versus D3 or FHBP alone) and therefore cellular maturation. The application of Met led to a dose-dependent inhibition of this maturation response, indeed the lowest concentration of Met $(1.25 \mathrm{mM})$ resulted in a modest, yet significant ${ }^{* *} \mathrm{p}<0.005$ compared to D3 \& FHBP) attenuation of the differentiation response. The application of $10 \mathrm{mM}$ Met gave a total ALP activity comparable to the control groups, i.e., complete inhibition. A similar effect was observed for AiCAR (Figure 1B) in which the treatment of MG63s with $1.25 \mathrm{mM}$ AiCAR significantly ( $\left.{ }^{*} \mathrm{p}<0.0001\right)$ blocked the differentiation of osteoblasts to co-stimulation with FHBP and D3. 
Figure 1A

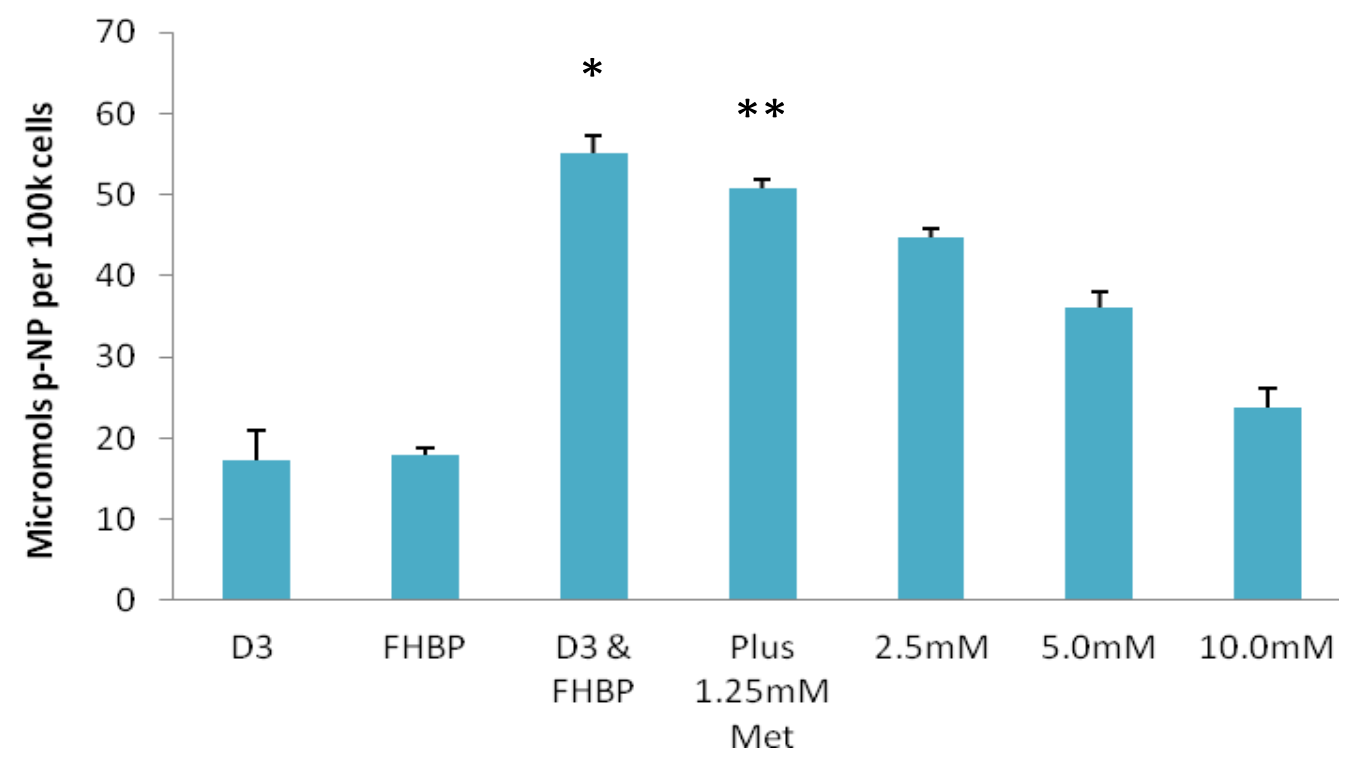

Figure 1B

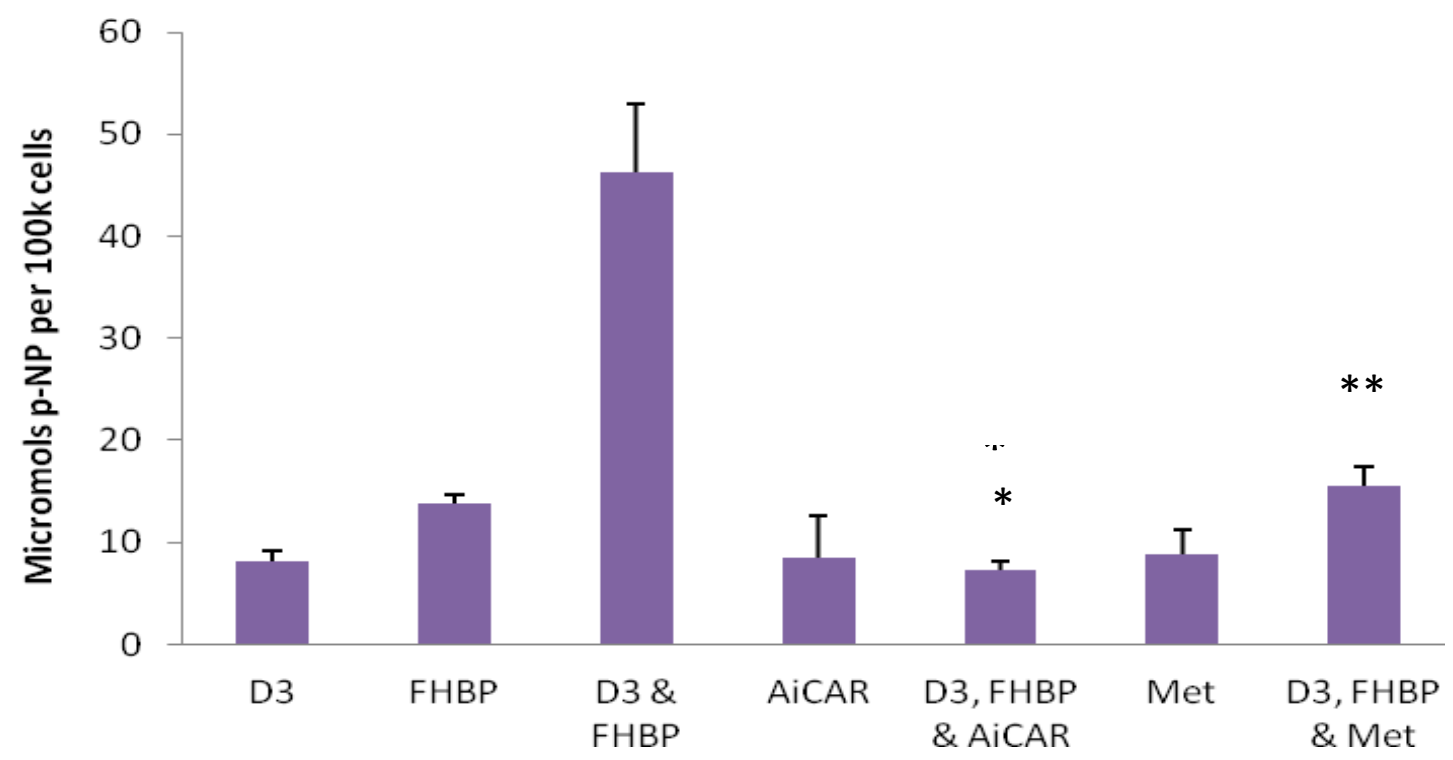

Figure 1: Metformin and AiCAR inhibit MG63 maturation: A-Cells were seeded into 24-well plates, allowed to reach confluency over a three day period and subsequently starved for 24 hours. MG63s were then co-treated with D3 (100nM) and the LPA1/3 receptor agonist, FHBP (500nM) to promote their maturation. The significant $\left({ }^{*} \mathrm{p}<0.0001\right.$ versus D3 and FHBP alone), synergistic increase in para-nitrophenol (p-NP), from p-NP phosphate, reflects greater ALP activity and therefore cellular maturation. This maturation event was inhibited when the cells were co-exposed to metformin (Met). Although modest, the effect of $1.25 \mathrm{mM}$ Met was able to significantly $\left({ }^{* *} \mathrm{p}<0.005\right)$ attenuate the maturation of MG63 to a combination of D3 and FHBP. Increasing the concentration of Met afforded greater inhibition of the maturation response. B-Similar results were obtained with AiCAR (1.25mM); AiCAR activates AMPK by serving as an AMP mimic [22] and was therefore used alongside Met (10mM) as an additional, structurally distinct, AMPK activation control. The data are expressed as the mean micromolar concentration of p-NP per 100k cells + SD.

\section{The highly potent and selective AMPK activator kills MG63 cells; rescue with compound C}

Unlike Met and AiCAR, the cell permeable AMPK activator A769662 (50 $\mu \mathrm{M})$ kills MG63 cells within 48 hours of exposure (Figure 2). To corroborate that the action of A769662 was via activation of AMPK, MG63 cells were pre-treated with compound C ( $5 \mu M)$, an AMPK inhibitor, for an hour and for the duration of A769662 exposure. The light micrographs (Figure 2A) presented clearly depict the demise of MG63 cells to A769662 which could be prevented with compound C. To gain greater insight of cell morphology in response to A769662, MG63 cells were seeded into the wells of chamber slides, treated over a 48 hour period and processed for fluorescence microscopy to visualise the F-actin (Alexa Fluor $594^{\mathrm{R}}$ ) and nuclear (DAPI) compartments (Figure 3, scale bar $50 \mu \mathrm{m}$ ).

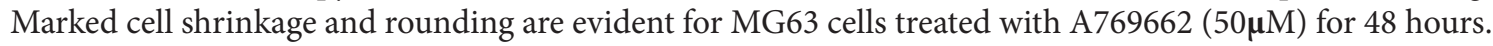




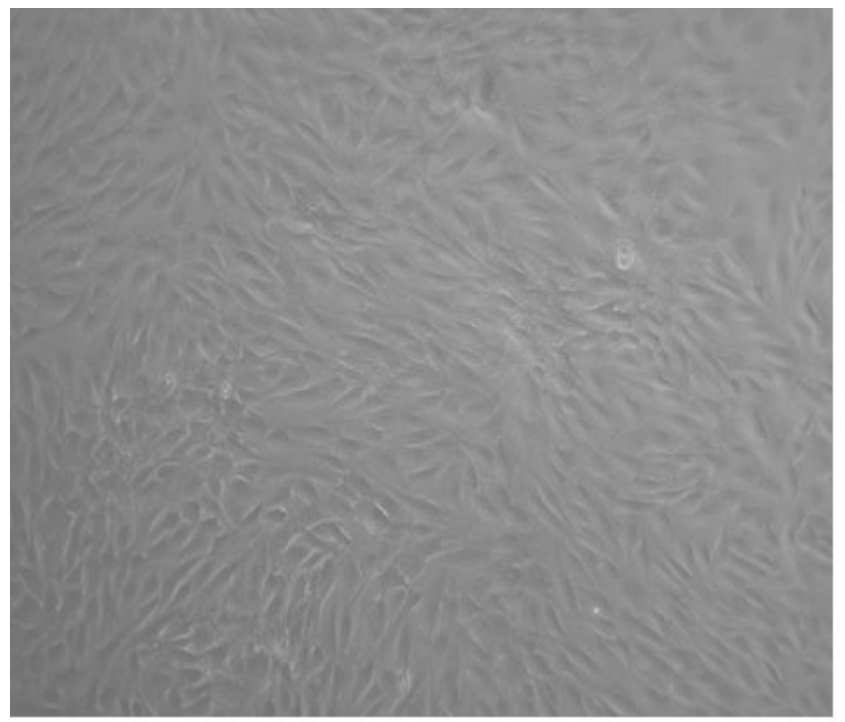

Vehicle control

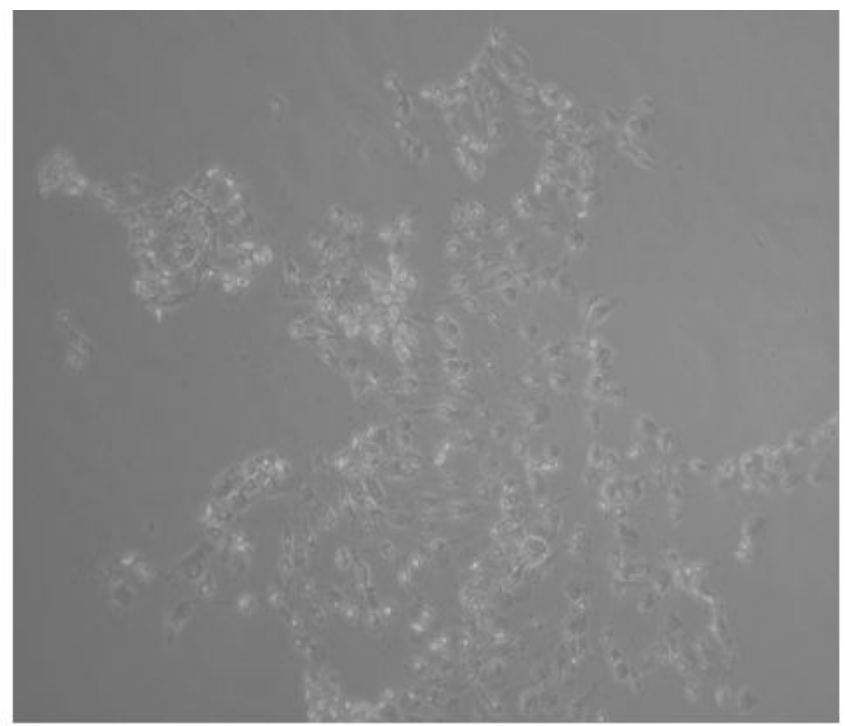

$50 \mu \mathrm{M}$ A769662

Figure 2A

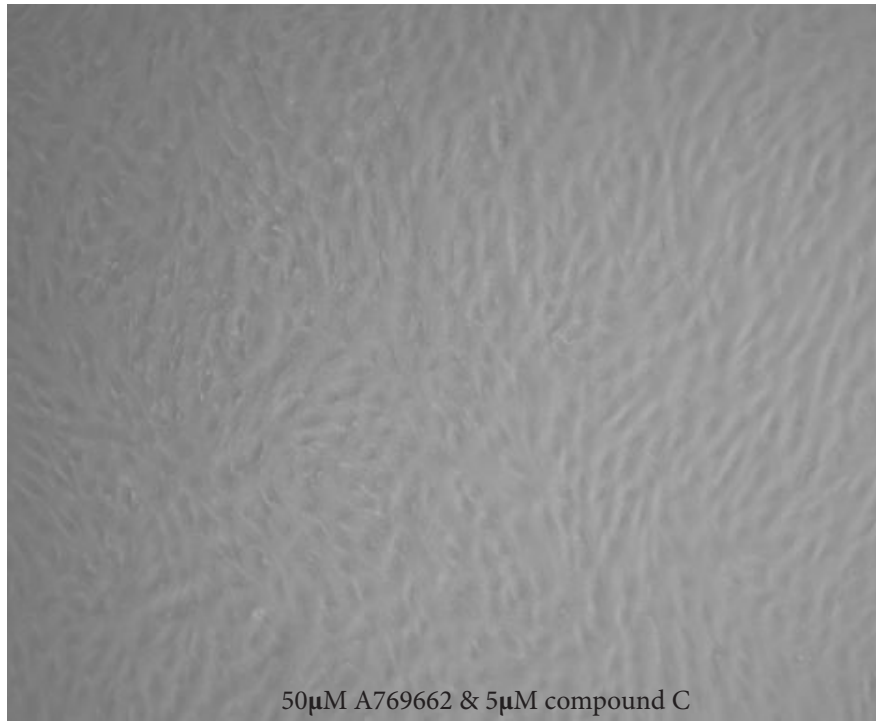

Figure 2B

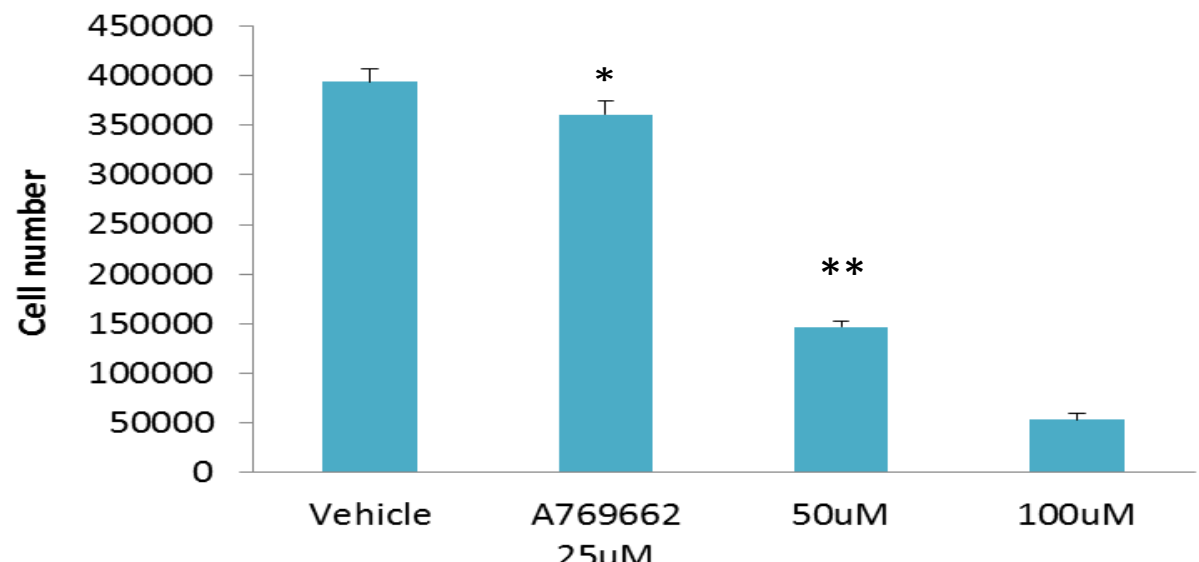

Figure 2: The cell permeable, selective AMPK activator, A769662, kills MG63 cells: A - MG63 cells were seeded into 24-well plates, allowed to reach confluency ( 3 days), starved for 24 hours and then treated with either serum free culture medium (vehicle control) or 50 $\mu \mathrm{M}$ A769662. Cells were left for 48 hours and photographs taken with a digital camera fitted to an inverted microscope. The images clearly depict cell demise on receipt of A769662. To corroborate that A769662-induced cell loss was via AMPK activation, MG63 cells were exposed to this thienopyridone in the presence of Compound C, a widely recognised AMPK inhibitor. The application of Compound C prevented cell losses in response to A769662 . B - Inset quantitative data pertaining to viable MG63 cell number in response to varying concentrations $(25-100 \mu \mathrm{M})$ of A769662 after a 48 hour culture period. Cell number was determined using MTS/PMS and formazan quantification at $490 \mathrm{~nm}$. Although there is a modest reduction in MG63 cell number ( $\mathrm{p}<0.05$ compared to the vehicle control) for cells treated with $25 \mu \mathrm{M}$ thienopyridone the number of cells are demonstrably reduced by approximately 2.5 and 8 -fold (" $\mathrm{p}<0.001$ compared to the vehicle control) when using the thienopyridone at 50 and $100 \mu \mathrm{M}$ respectively. 
Figure 3

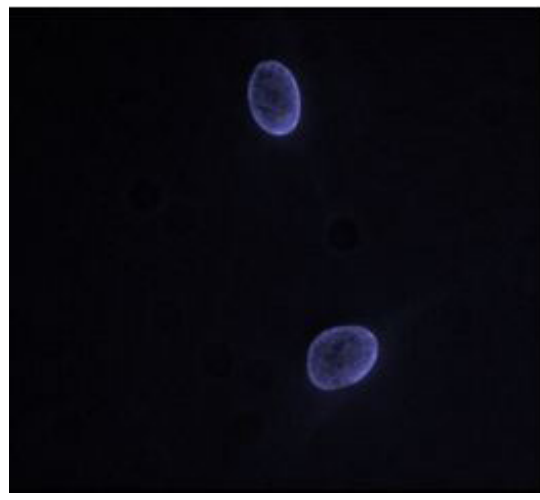

Veh $24 \mathrm{hr}$ - Nuclei

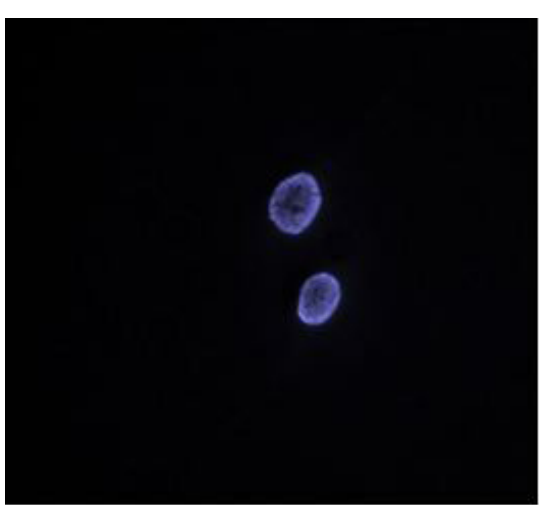

$50 \mu \mathrm{M}$ A769662

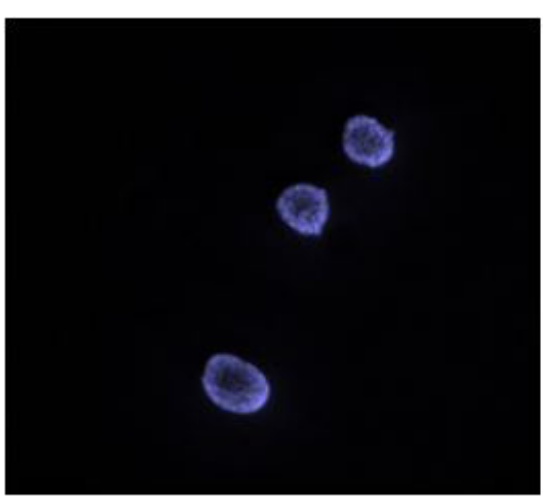

Veh $48 \mathrm{hr}$
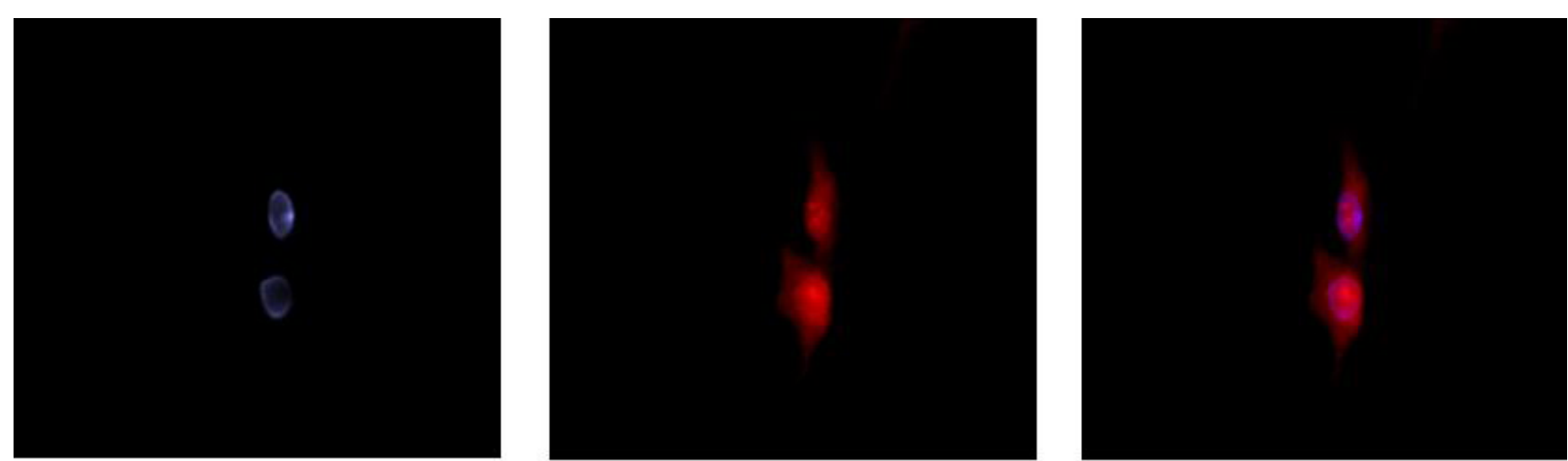

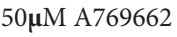

Figure 3: Temporal changes to MG63 morphology in response to A769662: To gain greater insight of cell morphology in response to A769662, MG63 cells were seeded into the wells of chamber slides, treated for 24 and 48 hours and processed for fluorescence microscopy to visualise the F-actin (Alexa Fluor $594^{\mathrm{R}}$ )

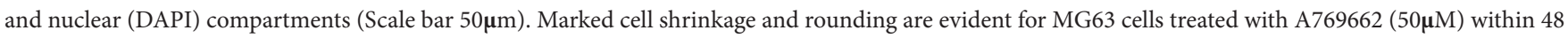
hours. 
Osteosarcoma-derived osteoblasts are susceptible to A769662 whereas primary human osteoblasts are not

The AMPK activator, A769662 $(100 \mu \mathrm{M})$ was applied to osteosarcoma sourced MG63 and Saos-2 cells and their growth response compared to primary human osteoblasts (hOBs) and their stem cell progenitors (hBMSC). Of the cell types examined only the MG63 and Saos-2 cells had fewer cells following a 48 hour exposure of A769662 (Figure 4A). Interestingly the magnitude of the response between the two osteosarcoma cell lines was noticeably different with MG63s consistently having far fewer numbers ( $\mathrm{p}<0.001, \sim 6$ fold less versus vehicle control) after the treatment period. However the decline in Saos-2 cell number was still significantly less (" $\mathrm{p}<0.001)$ than the control group by approximately 1.5 fold. Using the MG63 as a model system, the loss of cells to A769662 $(100 \mu \mathrm{M})$ could be significantly attenuated $\left({ }^{*} \mathrm{p}<0.001\right)$ when the cells were co-treated with a cell permeable, broad spectrum caspase inhibitor, Q-VD $(10 \mu \mathrm{M})$. As anticipated phenformin (Phen, $5 \mathrm{mM})$ was highly toxic to the cells but the inclusion of Q-VD was unable to rescue MG63s from this agent (Figure 4B).

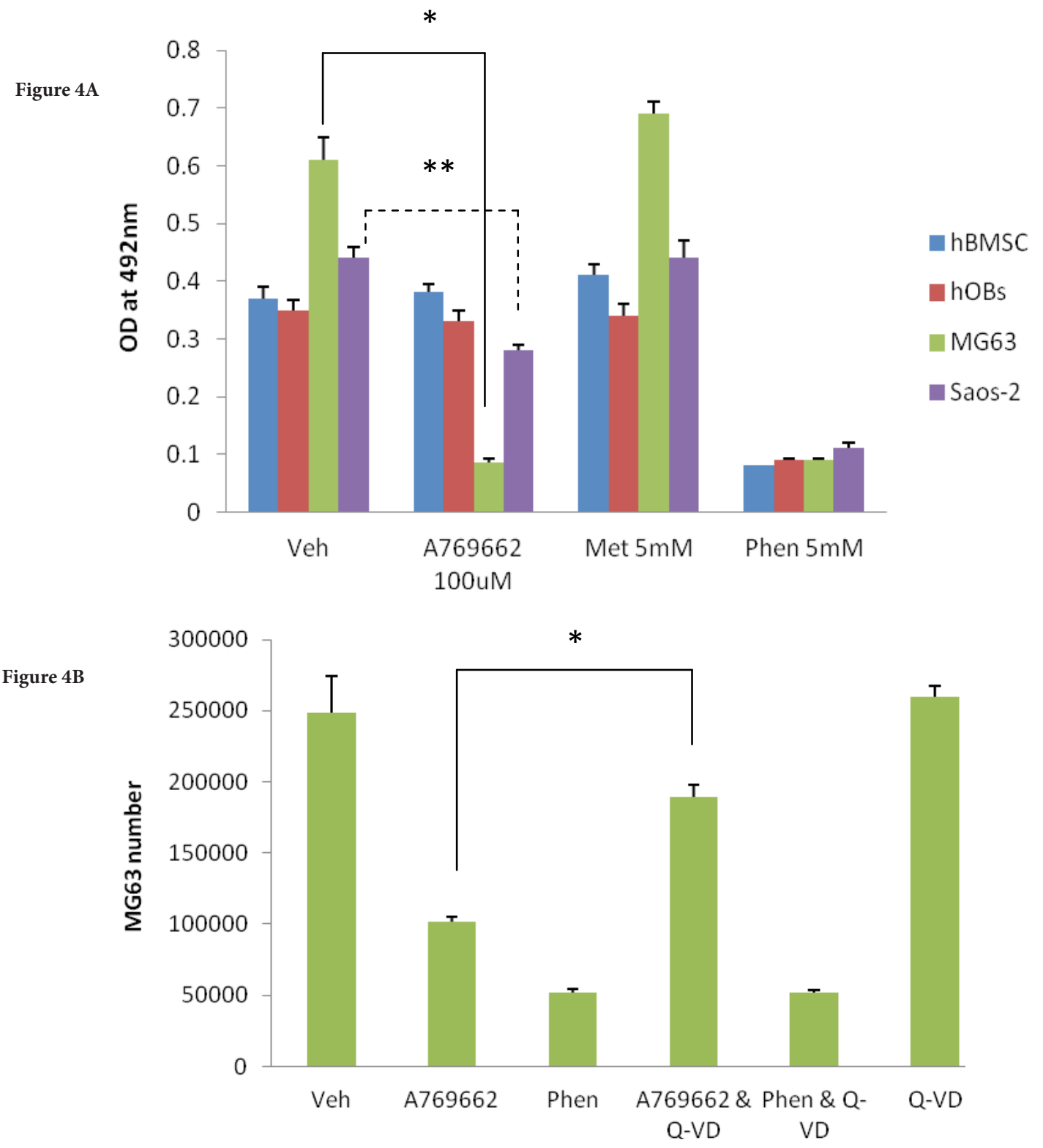

Figure 4: Osteosarcoma-derived osteoblasts are susceptible to the thienopyridone, A769662: A - Human bone marrow-derived stem (stromal) cells (hBMSCs), primary human osteoblasts (hOBs) and two p53 null osteosarcoma-derived cell lines (MG63 and Saos-2) were treated with equimolar (5mM) concentrations of either metformin (Met) or phenformin (Phen). These cells were also treated with the AMPK selective activator, A769662 (100 $\mu$ M). In each instance the cells were left for 48 hours and cell number assessed using MTS/PMS and formazan quantification at 490nm. As anticipated Phen was toxic to all cell types examined whereas Met was without influence on cell growth. Of the cell types examined only the MG63 and Saos-2 cells were susceptible to A769662; the magnitude of cell losses are noticeably different for these cells with MG63s experiencing greater reduction in number ( ${ }^{*}<0.001$ versus vehicle control). Although not as substantial, the Saos-2 cell number was found to be significantly less (** $\mathrm{p}<0.001$ than control cultures) when treated with A769662. B - Partial prevention of A769662-induced MG63 loss was achieved (*p<0.001 versus A769662 alone) using a pan caspase inhibitor (Q-VD, 10 $\mu$ M). This same inhibitor was unable to attenuate MG63 cell demise in response to phenformin (Phen). All data are expressed as the mean + SD and are representative from three independent experiments. 


\section{Discussion}

Clinically Met has a proven track record in the management of type 2 diabetes and there is increasing evidence that this drug could also be a valuable tool in the arsenal against a wide range of cancers [23,24]. In recent years a few independent studies have emerged reporting on the potentially beneficial role of Met in supporting pro-anabolic effects on bone forming osteoblasts. Collectively these works were conducted for either murine or rodent osteoblasts [4-7]. In addition there is evidence that Met blunts osteopaenia in rats following bilateral ovariectomy [8]. Whether Met exerts a direct effect on indices of bone metabolism is hard to say but it is worth mentioning that Met has been found to inhibit the secretion of GnRH from neurones (GT1-7 cells) and, in vivo, Met shortens the inter-oestrus interval in mice [25]. Given that elevated FSH stimulates osteoclastic bone resorption [26], compounds that block GnRH secretion might reduce the osteopaenia consequent to ovariectomy where the levels of FSH will be elevated. There is however one study that identifies a negative effect of Met on murine and rodent osteoblast maturation [27].

Since the search for existing drugs to treat/manage bone loss or indeed stimulate bone accrual for skeletal repair is ongoing it is especially important that studies using human osteoblasts be performed. To date the only study reporting on the effect of Met on human osteoblasts is a study by Takatani and colleagues [28] in this particular instance research addressed the influence of Met on the Wnt/ $\beta$ —catenin signalling pathway which plays an important role in osteoblast biology. The application of Met inhibited lithium chloride -induced Wnt transactivation. Similarly Met suppressed Wnt3a-induced transcriptional activity of T-cell transcription factor 1. Collectively these findings indicate that increasing AMPK activity in human osteoblasts could actually lead to a decline in bone mass through a reduction in osteoblast $\beta$-catenin levels. In our hands we find that Met does not stimulate the proliferation of MG63 osteoblast-like cells, primary human osteoblasts or their mesenchymal stem cell progenitors. Since the studies using murine and rodent osteoblasts indicated that Met might support cellular maturation we extended our studies to an investigation of Met on total ALP expression, a protein absolutely essential for bone collagen calcification [29] and a reliable indicator of osteoblast maturation. Suffice it to say Met, even as high as $10 \mathrm{mM}$, was unable to evoke any increase in total ALP, either alone or in concert with calcitriol, the active metabolite of D3 vital for securing healthy bone tissue formation [30]. On the contrary, Met actually blunted the ability of D3 and an LPA receptor agonist (FHBP) to induce osteoblast maturation, a robust agent pairing in promoting human osteoblast differentiation [31,32].

Our findings are therefore in agreement with the study of Takatani and colleagues [28] for human Saos-2 cells where AMPK activation actually attenuates responses conducive to promoting cellular maturation. Our findings also agree with those of Kasai and colleagues [27] where the application of Met to murine osteoblasts inhibited matrix calcification in extended cell cultures. Although prior published works, albeit for non-human osteoblasts, suggested Met might be beneficial to bone, our own findings are to the contrary. As with our findings reported herein, the studies of Kasai and co-workers [27] and Takatani et al. [28] report that millimolar concentrations of Met are required to prosecute the observed responses which probably indicates a lack or low expression of organic cation transporters (OCTs) which are required for the carriage of Met into cells. Indeed there is compelling evidence that OCT1 modulates AMPK activation [33]. Furthermore the abundant expression of OCT1 by hepatic cells is the likely explanation for why Met is able to lower blood glucose and why the liver accumulates Met at several hundred micromolar in the periportal area [24]. Because OCTs are required for Met carriage it is not surprising therefore that the majority of in vitro studies report on the biological effects of Met in the millimolar range.

With regard to the aforementioned murine/rodent studies it is the collected belief that Met brings about the changes observed via activation of AMPK. Although Met is unable to activate the enzyme in a cell free system [34] Met does, however, target complex I of the mitochondrial respiratory chain which would mean indirect AMPK activation consequent to reduced ATP but raised AMP. i.e., a reduction in cellular energy charge [35]. Complex I is critical for reduced NADH to maintain the mitochondrial proton gradient necessary for ATP production, therefore inhibition of complex I, by Met, leads to a reduction in overall cellular energy charge [10]. This would seem at odds with a pro-anabolic action of Met reported for murine and rodent osteoblasts; AMPK activation in response to Met would lead to a decline of energy consumption, i.e., anabolism, with a paralleled increase in energy production [36] and there is certainly evidence that Met can inhibit collagen synthesis in human hepatic stellate cells [37]. It is unlikely therefore that AMPK-activating agents, including Met, will promote the synthesis of bone matrix, a composite of type I collagen impregnated with hydroxyapatite.

As with so many other studies we had to administer Met to human osteoblasts in the millimolar range to track any changes in growth or cellular maturation. High concentrations of the drug may have stimulated "off target" effects and so to more reliably target AMPK we considered the use of two other cell permeable agonists of this kinase to see what influence these compounds might have on osteoblast growth and to extend these studies to primary human osteoblasts and their stem cell progenitors. In the first instance we used phenformin, a Met analogue known to partition into cells independent of OCTs [38]. In addition we used A769662, a novel thienopyridone which directly acivates AMPK [11,39,40] in a manner distinct from AMP [41]. These two agents gave very different responses; phenformin was toxic to all the cell types examined, an event that could not be prevented or attenuated using compound C, a known antagonist of AMPK [42] or the broad spectrum caspase inhibitor, Q-VD (data not shown). However, A769662 was only toxic to MG63 osteoblasts, albeit from 50 $\mu$ M.

The effect of A769662 on MG63 cell death could be prevented by co-treating the cells with compound C. Furthermore, the application Q-VD was able to attenuate A769662-induced cell death. These findings suggested that the activation of AMPK by 
A769662 actually precipitated the demise of a cell type known to be negative for the tumor suppressor, p53 [43]. MG63 cells are an osteoblast-like cell sourced from an osteosarcoma, and silencing p53 is necessary for the development of these tumours [44]. Interestingly cells deficient in p53 are especially vulnerable to AMPK activation. Indeed there is very good evidence to indicate that the activation of AMPK is selectively toxic to cells negative for p53 [45]. Similarly Saos-2 cells were also susceptible to A769662, although the magnitude of the response was clearly less than that observed for MG63s. Paradoxically, loss of p53 affords some resistance to stressful situations such as nutrient withdrawal and hypoxia. As such these aberrant cells are able to thrive where healthy cell types might perish. However there is compelling evidence that activation of AMPK in p53-negative cells brings about a metabolic scenario that the cells are unable to tolerate which ultimately culminates in their demise [45].

Our findings are certainly in agreement with those of Buzzai and colleagues [45] in that AMPK activation in p53-null osteosarcomaderived osteoblasts promotes cell death. However the finding that Met is without effect probably reflects little or no expression of OCTs. Therefore cell-permeable compounds known to activate AMPK are much more likely to become fruitful adjuncts to the treatment and management of osteosarcomas which are widely known to be a consequence of p53 silencing [44,46]. Although initially tempting that Met might find a place in bone repair this biguanide does not promote the growth and/or maturation of human osteoblasts. In fact Met exhibited an inhibitory effect on D3-induced cellular maturation but only when applied at high concentrations in the millimolar range. However a molecule that shares its ability with Met to activate AMPK, namely A769662, should be considered in the fight to eradicate osteosaroma or indeed other tumour cell types deficient in p53/function.

\section{Acknowledgement}

The corresponding author is indebted to the Musculoskeletal Research Unit (MRU) for the financial support to conduct the research described herein. All authors would like to thank Miss Anna Rogers (MRU) for her invaluable assistance with capturing the fluorescence microscopy images depicted. Each of the authors declares no conflicts of interest.

\section{References}

1. Ashburn TT and Thor KB (2004) Drug repositioning: identifying and developing new uses for existing drugs. Nat Rev Drug Discov 3: 673-83.

2. Chong C, Sullivan JD (2007) New uses for old drugs. Nature 448: 645-6.

3. Witters LA (2001) The blooming of the French lilac. J Clin lnvest 108: 1105-7.

4. Cortizo AM, Sedlinsky C, McCarthy AD, Blanco A, Schurman L (2006) Osteogenic actions of the anti-diabetic drug metformin on osteoblasts in culture. Eur J Pharmacol 536: 38-46.

5. Jang WG, Kim EJ, Bae IH, Lee KN, Kim YD, et al. (2011) Metformin induces osteoblast differentiation via orphan nuclear receptor SHP-mediated transactivation of Runx2. Bone 48: 885-93.

6. Kanazawa I, Yamaguchi T, Yano S, Yamauchi M, Sugimoto T (2008) Metformin enhances the differentiation and mineralization of osteoblastic MC3T3-E1 cells via AMP kinase activation as well as eNOS and BMP-2 expression. Biochem Biophys Res Commun 375: 414-9.

7. Shah M, Kola B, Bataveljic A, Arnett TR, Viollet B, et al. (2010) AMP-activated protein kinase (AMPK) activation regulates in vitro bone formation and bone mass. Bone 47: 309-19.

8. Gao Y, Li Y, Xue J, Jia Y, Hu J (2010) Effect of the anti-diabetic drug metformin on bone mass in ovariectomized rats. Eur J Pharmacol 635: 231-6.

9. Molinuevo MS, Schurman L, McCarthy AD, Cortizo AM, Tolosa MJ, et al. (2010) Effect of metformin on bone marrow progenitor cell differentiation: in vivo and in vitro studies. J Bone Miner Res 25: 211-21.

10. Miller RA, Birnbaum MJ (2010) An energetic tale of AMPK-independent effects of metformin. J Clin Invest 120: 2267-70.

11. Scott JW, van Denderen BJ, Jorgensen SB, Honeyman JE, Steinberg GR, et al. (2008) Thienopyridone drugs are selective activators of AMP-activated protein kinase beta1-containing complexes. Chem Biol 15: 1220-30.

12. Winder WW, Hardie DG (1996) Inactivation of acetyl-CoA carboxylase and activation of AMP-activated protein kinase in muscle during exercise. Am J Physiol 270: E299-304.

13. Kassi E, Papavassiliou AG (2008) A possible role of osteocalcin in the regulation of insulin secretion: human in vivo evidence? J Endocrinol 199: 151-3.

14. Ducy P, Desbois C, Boyce B, Pinero G, Story B, et al. (1996) Increased bone formation in osteocalcin-deficient mice. Nature 382: 448-52.

15. Reinehr T, Roth CL (2010) A new link between skeleton, obesity and insulin resistance: relationships between osteocalcin, leptin and insulin resistance in obese children before and after weight loss. Int J Obes (Lond) 34: 852-8.

16. Ferron M, Hinoi E, Karsenty G, Ducy P (2008) Osteocalcin differentially regulates beta cell and adipocyte gene expression and affects the development of metabolic diseases in wild-type mice. Proc Natl Acad Sci U S A 105: 5266-70.

17. Clemens TL, Karsenty G (2011) The osteoblast: An insulin target cell controlling glucose homeostasis. J Bone Miner Res 26: 677-80.

18. Ducy P (2011) The role of osteocalcin in the endocrine cross-talk between bone remodelling and energy metabolism. Diabetologia 54: 1291-7.

19. Gidley J, Openshaw S, Pring ET, Sale S, Mansell JP (2006) Lysophosphatidic acid cooperates with 1alpha,25(OH)2D3 in stimulating human MG63 osteoblast maturation. Prostaglandins Other Lipid Mediators 80: 46-61.

20. Mansell JP, Barbour M, Moore C, Nowghani, Pabbruwe M, et al. (2010) The synergistic effects of lysophosphatidic acid receptor agonists and calcitriol on MG63 osteoblast maturation at titanium and hydroxyapatite surfaces. Biomaterials 31: 199-206.

21. Mansell JP, Nowghani M, Pabbruwe M, Paterson IC, Smith AJ, et al. (2011) Lysophosphatidic acid and calcitriol co-operate to promote human osteoblastogenesis: Requirement of albumin-bound LPA. Prostaglandins ther Lipid Mediat 95: 45-52. 
22. Corton JM, Gillespie JG, Hawley SA, Hardie DG (1995) 5-aminoimidazole-4-carboxamide ribonucleoside. A specific method for activating AMP-activated protein kinase in intact cells? Eur J Biochem 229: 558-65.

23. Kourelis TV, Siegel RD (2012) Metformin and cancer: new applications for an old drug. Med Oncol 29: $1314-27$.

24. Viollet B, Guigas B, Sanz Garcia N, Leclerc J, Foretz M, et al. (2012) Cellular and molecular mechanisms of metformin: an overview. Clin Sci (Lond) 122: 253-70. 25. Menassol JB, Tautou C, Collet A, Chesneau D, Didier Lomet, et al. (2011) The effect of an intracerebroventricular injection of metformin or AICAR on the plasma concentrations of melatonin in the ewe: potential involvement of AMPK? BMC Neurosci 12: 76.

26. Sun L, Peng Y, Sharrow AC, Iqbal J, Zhang Z, et al. (2006) FSH Directly Regulates Bone Mass. Cell 125: 247-60.

27. Kasai T, Bandow K, Suzuki H, Chiba N, Kakimoto K, et al. (2009) Osteoblast differentiation is functionally associated with decreased AMP kinase activity. J Cell Physiol 221: 740-9.

28. Takatani T, Minagawa M, Takatani R, Kinoshita K, Kohno Y (2011) AMP-activated protein kinase attenuates Wnt/ $\beta$-catenin signaling in human osteoblastic Saos-2 cells. Mol Cell Endocrinol 339: 114-9.

29. Whyte MP (2010) Physiological role of alkaline phosphatase explored in hypophosphatasia. Ann N Y Acad Sci 1192: 190-200.

30. Sharma S, Barr AB, Macdonald HM, Sheehy T, Novotny R, et al. (2011) Vitamin D deficiency and disease risk among aboriginal Arctic populations. Nutr Rev 69: 468-78.

31. Blackburn J, Mansell JP (2012) The emerging role of lysophosphatidic acid (LPA) in skeletal biology. Bone 50: 756-62.

32. Lancaster ST, Blackburn J, Blom A, Makashima M, Ishizawa M, et al. (2014) 24,25-Dihydroxyvitamin D3 cooperates with a stable, fluoromethylene LPA receptor agonist to secure human (MG63) osteoblast maturation. Steroids 83: 52-61.

33. ShuY, Sheardown S, Brown C, Owen R, Zhang S, et al. (2007) Effect of genetic variation in the organic cation transporter 1 (OCT1) on metformin action. J Clini lnvest 117: 1422-31.

34. Hawley SA, Gadalis A, Skytte Olsen G, Hardie DG (2002) The antidiabetic drug metformin activates the AMP-activated protein kinase cascade via an adenine nucleotide-independent mechanism. Diabetes 51: 2420-5.

35. Owen M, Doran E, Halestrap A (2000) Evidence that metformin exerts its anti-diabetic effects through inhibition of complex 1 of the mitrochondrial respiratory chain. Biochem J 348: 607-14.

36. Steinberg GR, Kemp BE (2009) AMPK in Health and Disease. Physiol Rev 89: 1025-78.

37. Lim JY, Oh MA, Kim WH, Sohn HY, Park SI (2011) AMP-activated protein kinase inhibits TGF-beta-induced fibrogenic responses of hepatic stellate cells by targeting transcriptional coactivator p300. J Cell Physiol 227: 1081-9.

38. Hawley SA, Ross FA, Chevtzoff C, Green KA, Evans A, et al. (2010) Use of cells expressing y subunit variants to identify diverse mechanisms of AMPK activation. Cell Metab 11: 554-65.

39. Cool B, Zinker B, Chiou W, Kifle L, Cao N, et al. (2006) Identification and characterization of a small molecule AMPK activator that treats key components of type 2 diabetes and the metabolic syndrome. Cell Metab 3: 403-16.

40. Goransson O, McBride A, Hawley SA, Ross FA, Shpiro N, et al. (2007) Mechanism of action of A-769662, a valuable tool for activation of AMP-activated protein kinase. J Biol Chem. 282: 32549-60.

41. Sanders MJ, Ali ZS, Hegarty BD, Heath R, Snowden MA, et al. (2007) Defining the mechanism of activation of AMP-activated protein kinase by the small molecule A-769662, a member of the thienopyridone family. J Biol Chem 282: 32539-48

42. Zhou G, Myers R, Li Y, Chen Y, Shen X, et al. (2001) Role of AMP-activated protein kinase in mechanism of metformin action. J Clin Invest 108: 1167-74.

43. Masuda H, Miller C, Koeffler H, Battifora H, Cline MJ (1987) Rearrangement of the p53 gene in human osteogenic sarcomas. Proc Natl Acad Sci U S A 84: 7716-9.

44. Jones KB (2011) Osteosarcomagenesis: modeling cancer initiation in the mouse. Sarcoma 694136.

45. Buzzai M, Jones RG, Amaravadi RK, Lum JJ, DeBerardinis RJ, et al. (2007) Systemic treatment with the antidiabetic drug metformin selectively impairs p53-deficient tumor cell growth. Cancer Res 67: 6745-52.

46. Lengner C J, Steinman HA, Gagnon J, Smith TW, Henderson JE, et al. (2006) Osteoblast differentiation and skeletal development are regulated by Mdm2-p53 signaling. JCB 172: 909-21. 